\title{
ARTICLE OPEN \\ Inexpensive and flexible nanographene-based electrodes for ubiquitous electrocardiogram monitoring
}

\author{
Francisco J. Romero (iD ${ }^{1,2}$, Encarnacion Castillo ${ }^{2}$, Almudena Rivadeneyra ${ }^{1,2}$, Alejandro Toral-Lopez ${ }^{1,2}$, Markus Becherer ${ }^{3}$,
} Francisco G. Ruiz ${ }^{1,2}$, Noel Rodriguez ${ }^{1,2}$ and Diego P. Morales $\mathbb{1}^{2,4}$

Flexible electronics is one of the fundamental technologies for the development of electronic skin, implant wearables, or ubiquitous biosensing. In this context, graphene-derived materials have attracted great interest due to their unique properties to fulfill the demands of these applications. Here we report a simple one-step method for the fabrication of electrophysical electrodes based on the photothermal production of porous nanographene structures on the surface of flexible polyimide substrates. This approach constitutes an inexpensive alternative to the commercial medical electrodes, leading to a lower and much more stable skin-electrode contact resistance and providing comparable signal transduction. This technology has been framed inside the loT paradigm through the development of a denoising and signal classification clustering algorithm suitable for its implementation in wearable devices. The experiments have shown promising achievements regarding noise reduction, increasing the crest factor $\sim 3.7 \mathrm{~dB}$, as well as for the over $90 \%$ heart rate-monitoring accuracy.

npj Flexible Electronics (2019)3:12; https://doi.org/10.1038/s41528-019-0056-2

\section{INTRODUCTION}

Cardiovascular diseases (CVDs) are the most common cause of death in the world. ${ }^{1}$ This has led to assign great efforts to their early diagnosis, prevention, and treatment. According to the European Heart Network (EHN), overall CVDs are estimated to cost the EU economy 210 billion a year, of which around 53\% (111 billion) are intended to the health costs. ${ }^{2,3}$ Electrocardiography (ECG) is the most common method to diagnose abnormalities in the cardiac activity and to extract the information that might reveal CVDs in advance. ${ }^{4}$ So far, this technique was traditionally limited to the hospital environment; however, the emergence of new technological paradigms, such as the Internet of Things (IoT), flexible electronic devices and electronic textiles (e-textiles) is shifting the "in-hospital care" towards the ubiquitous healthmonitoring. $^{5-7}$

Metals such as silver $(\mathrm{Ag})$ or copper $(\mathrm{Cu})$ are currently the most used materials for the biosignal transduction. ${ }^{8-10}$ There is no doubt that these materials have offered high performance in this kind of applications and have contributed to the process of medical diagnosis. However, wearable health-monitoring systems (WHMS) also require of inexpensive, lightweight, and biocompatible conductive materials. Following these premises, flexible electronics and carbon-based materials are a symbiosis expected to play an important role in the next generation of WHMS. ${ }^{1-14}$ Celik et al. presented an electrode fabricated by chemical vapor deposition coating of graphene on top of a Agbased electrode; ${ }^{15}$ chemically reduced graphene oxide on polyethylene terephthalate (PET) and porous textile substrates have been also studied by Lou et al. and Karim et al., respectively. ${ }^{16,17}$ Moreover, Jung et al. also proposed a carbonnanotube (CNT) on polydimethylsiloxane (PDMS) composite- based electrode for the ECG monitoring. ${ }^{18}$ All these proposed electrodes, in addition of being biocompatible, are suitable for long-term monitoring due to their "dry" nature, since they do not require of the use of adhesive or conductive gels, in contrast to the $\mathrm{Ag} / \mathrm{AgCl}$ ones. ${ }^{5}$ However, they are subjected to manufacturing processes with multiple stages, and therefore high production costs. In addition, the signal transduction capability of the electrodes must be good enough for the subsequent processing of the recorded signals. It is also important to keep in mind that wearables devices are subjected to significant restrictions of size, computational capabilities and power consumption. Therefore, it is necessary to ensure a compromise between both the raw biosignal quality and the optimization of the processing algorithms to obtain accurate results.

In this article, we propose the use of a low-power laser diode to produce a foam of porous graphene (PG) on a flexible substrate; this structure is applied to the fabrication of electrodes for the continuous and ubiquitous monitoring of ECG signals. As reported by previous works, PG with high electrical conductivity can be induced on the surface of diverse carbon-rich materials by a photothermal process. ${ }^{19}$ Recently, we presented an in-depth study of the laser-induction of PG on a flexible Kapton ${ }^{\circledR}$ substrate under ambient conditions by using a laser diode. ${ }^{20}$ This approach offers the advantage of defining high-precision conductive patterns of laser-induced nanographene aggregates (LINA) on flexible substrates without the need for lithographic masks or the deposition of any material before the laser treatment, such as occurs for the laser-reduced graphene oxide (laser-rGO). ${ }^{21}$ This one-step technique is also compatible with roll-to-roll methods, enabling an inexpensive mass-production of samples. The feasibility of the proposed electrodes for recording electrophysiological signals is

\footnotetext{
${ }^{1}$ Pervasive Electronics Advanced Research Laboratory, University of Granada, 18071 Granada, Spain; ${ }^{2}$ Department of Electronics and Computer Technology, University of

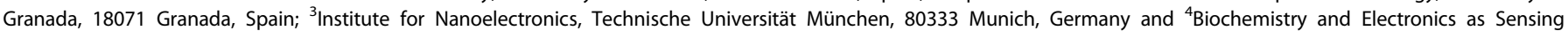
Technologies Group, University of Granada, 18071 Granada, Spain

Correspondence: Francisco J. Romero (franromero@ugr.es)
}

Received: 5 March 2019 Accepted: 26 May 2019

Published online: 14 June 2019 
demonstrated by monitoring the heart rate through a clustering algorithm intended to be implemented in portable hardware, such as wearables. This algorithm performs, on one side, the artifact removal from the ECG signal (baseline wandering (BW), tremor artifacts, etc.) and, on the other side, the extraction of the heart beat from the QRS-complexes by means of the classification of the peaks in different clusters. Thus, the classification is much more robust against noise and amplitude variations, since it is based on similarities instead of on fixed values as in the case of thresholdbased methods. The results have shown that the proposed electrodes, in combination with data analysis techniques, pave the way for the inexpensive and simple fabrication of electrodes for a new generation of wearable monitoring devices.

\section{RESULTS AND DISCUSSION}

\section{Electrode design and fabrication}

Figure 1a illustrates a schematic of the structure of the developed electrodes, fabricated following the basis of the laser-scribing procedure proposed in our previous work. ${ }^{20}$ The Kapton ${ }^{\circledR} \mathrm{HN}$ tape was cut into squares $(20 \mathrm{~mm} \times 20 \mathrm{~mm})$, then a circumference with a diameter of $10 \mathrm{~mm}$ was photothermally patterned on its surface using a computer numerical control (CNC)-driven laser diode. The electrode was laser-scribed at a fluence of $20 \mathrm{~J} \mathrm{~cm}^{-2}$ (laser spot diameter: $20 \mu \mathrm{m}$ ) and at an excursion rate of $3 \mathrm{~min} \mathrm{~cm}^{-2}$ in two perpendicular passes (one for $X$-axis and one for $Y$-axis), ensuring the carbonization process and the integrity of the substrate in terms of thermal dissipation. ${ }^{22}$ Next, a planar wire was connected to the LINA electrode using Ag-based conductive paint. This electrical connection approach provides a low contact resistance due to the increase of the contact area resulting from the high porosity of the nanographene-induced patterns. ${ }^{20}$ Most common commercial electrodes are based on $\mathrm{Ag} / \mathrm{AgCl}$ composites, being the surface of contact between the skin and the electrode of $78.5 \mathrm{~mm}^{2}$. Therefore, the whole structure was isolated using laminating pouches (as shown in Fig. 1a) leaving the access to the LINA circumference free in order to establish an appropriate comparison between our electrodes and the commercial ones (Supplementary Fig. S1). An adhesive skin patch with a transparent polyurethane (PU) film and a polyacrylate adhesive was used to attach the electrodes safely on the skin, as shown in Fig. $1 \mathrm{~b}$.

\section{Electrode characterization}

A SEM image of the patterned area of the flexible polyimide substrate is shown in Fig. 1c. As can be observed, the laserinduced nanographene patterns show a mesh-like structure as a consequence of the mechanical resolution of the CNC unit $(\sim 40 \mu \mathrm{m})$. Besides, the ablated surface (non-bright areas) exhibits a high porosity, which is a distinctive feature of the laser-induced porous nanographene. ${ }^{23}$ The LINA increases substantially the porosity of the polyimide, resulting in an increase of the thickness of $18.7 \pm 2.2 \mu \mathrm{m}$ over its surface, which enhances the overall contact interface. The nanographene nature of the induced patterns is confirmed by the Raman spectrum (Fig. 1d), which is composed of three main peaks ( $D$ peak: $\sim 135 \mathrm{~cm}^{-1}, \mathrm{G}$ peak: $\sim 158 \mathrm{~cm}^{-1}$, 2D peak: $\left.\sim 270 \mathrm{~cm}^{-1}\right)$. However, it is far from achieving the single-layer pristine graphene structure, since the ratio $I_{\mathrm{D}} / I_{\mathrm{G}} \simeq$ 1 indicates that the $s p^{2}$-hybridized carbonous systems, which compose the surface present defects in their crystalline structure, while the $I_{2 D} / I_{G}$ ratio reflects the $3 \mathrm{D}$ nature of $P G .{ }^{24}$ Furthermore, XPS results demonstrate the structure alterations induced by the photothermal process (Supplementary Fig. S2). The initial relative content of carbon $(\sim 75 \%)$, present as $\mathrm{C}-\mathrm{C} s p^{3}$ hybridized bonds, is increased up to $\sim 85 \%$ after the laser ablation, more than a half corresponding to $\mathrm{C}-\mathrm{C} s p^{2}$ hybridized carbon. The increase of the relative content of carbon is the outcome of the release as gases of the nitrogen and oxygen from the polyimide substrate. Thus, both oxygen and nitrogen relative contents are reduced by more than $10 \%$ and $5 \%$, respectively, as a result of the photothermal process. In addition to the chemical alterations, the LINA-induced patterns also present a great enhancement of the electrical conductivity with respect to the precursor substrate. The four-wire sensing experiments demonstrate that, through the photothermal a

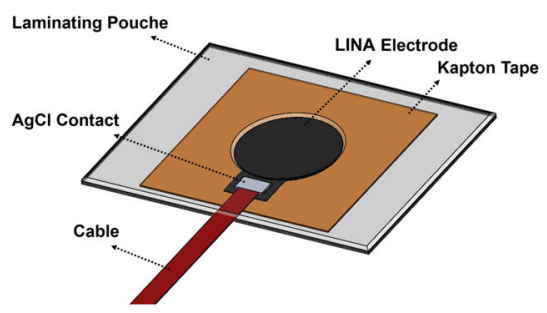

b

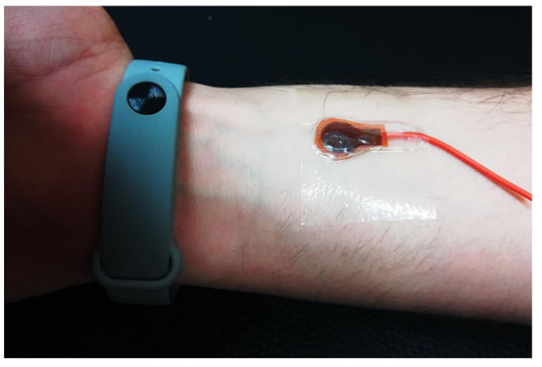

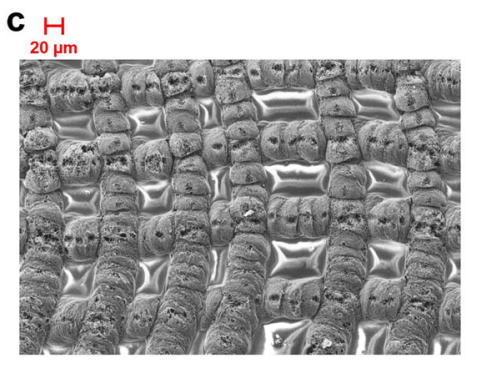

d
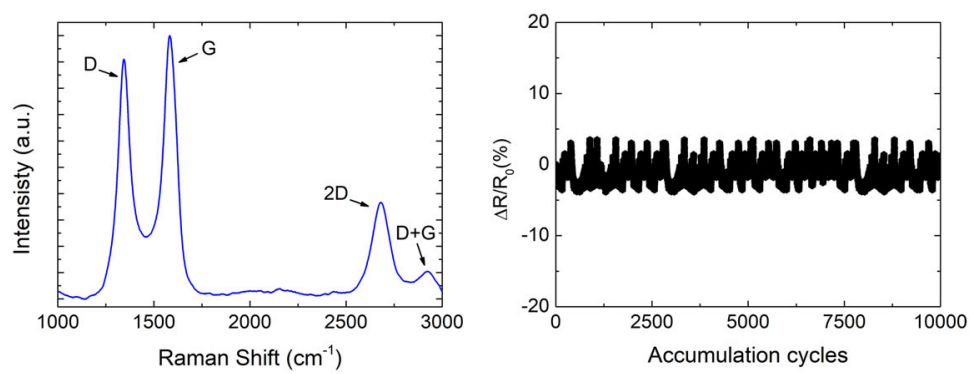

Fig. 1 Electrode fabrication and material characterization. a Schematic of the flexible electrode. $\mathbf{b}$ Flexible electrode attached to the forearm of a person. c SEM-image of the laser patterned surface (scale bar: $20 \mu \mathrm{m}$ ). The bright areas corresponds to the non-irradiated surface, while the dark ones are that of laser-induced nanographene aggregate. $\mathbf{d}$ Raman spectrum of the laser-induced porous nanographene aggregates. e Percentage of change in resistance $\left(R / R_{0}(\%)\right)$ for a increasing number of bending cycles, being $R_{0}$ the initial resistance and $R$ the resistance measured after each bending cycle 
ablation process, the sheet resistance of the samples can be reduced down to values closed to $250 \Omega \square^{-1}$. The bending dependence of the sheet resistance was also investigated to demonstrate the feasibility of the flexible electrodes for a longterm monitoring. Figure 1e shows the normalized change in the resistance of an electrode $\left(\Delta R / R_{0}(\%)\right)$ as a function of the bending cycles accumulated. The results have confirmed that the difference in the electrode resistance between the initial state and the state after $10^{4}$ bending cycles are negligible $(<4 \%)$. Further, we compared the skin-electrode contact impedance of the fabricated electrodes with two different commercial wet electrodes (see the section "Materials") aiming to compare their frequency response, which is normally modeled as the association of parallel connections of resistors and capacitors. ${ }^{25}$ Results have shown promising values of skin-electrode impedance as a function of the frequency when compared with the commercial electrodes, as depicted in Fig. 2a. On one hand, the commercial electrodes show a contact impedance more than 15 times higher than that of the electrodes presented in this work at low frequencies $(\sim 1 \mathrm{k} \Omega)$. On the other hand, the frequency response of the contact impedance of the LINA electrodes stands out for its lower ratio of change in frequency $\left(2.33 \Omega \mathrm{Hz}^{-1}\right)$, while the commercial electrodes present a deviation with respect to the frequency of 66.52 and $37.84 \Omega \mathrm{Hz}^{-1}$, respectively, due to the higher impact of the parasitic capacitance when the frequency increases. The notable achievement of the LINA electrodes is attributed to the high porosity of the material, which enhances the specific contact area of the interface skin-electrode decreasing both the resistance and the capacitance seen from the body to the electrodes.

ECG and heart rate monitoring with LINA electrodes The raw signals recorded from both the commercial and the flexible electrodes developed are comparable in terms of amplitude and signal-to-noise ratio. Figure $2 \mathrm{~b}$ shows an extract of $3.5 \mathrm{~s}$ from two simultaneous ECG recordings where the $\mathrm{P}-\mathrm{QRS}-\mathrm{T}$ complexes can be clearly distinguished. ${ }^{26}$ The measuring electrodes were placed on a volunteer according to the Lead I Einthoven's triangle scheme. ${ }^{27}$ Thus, the positive electrode was placed on the left wrist, the negative electrode was placed on the right wrist and the reference one on the left ankle. Under the configurations parameters of the ECG acquisition system (see the section "Materials and methods"), the signals present an average R-peak amplitude of $\sim 467 \mathrm{mV}$ with a crest factor (peak-to-RMS ratio) of 11.40 and $11.47 \mathrm{~dB}$ for $\mathrm{Ag}$-based electrodes and LINA ones, respectively. The feasibility of the proposed electrodes for the WHMS paradigm is further demonstrated combining them with an algorithm that can be applied not only to filter and preprocessing the signal obtained from the LINA electrodes but also to extract the heart rate. ${ }^{28}$ This algorithm can be divided in two main blocks, as schematized in Fig. 2c, d. First, we apply a modified version of the discrete wavelet transform (DWT) optimized for portable hardware implementations to reduce the BW and noise of the recorded ECG signals produced by the motion artifacts (see the section "Materials and methods"). The resulting signal after the baseline wander correction and noise suppression is shown in Fig. 3b, where the crest factor has been increased up to $15.24 \mathrm{~dB}$. After that, a clustering-based method, depicted in Fig. $2 d$ and described in the section "Materials and methods", is applied to the pre-processed signal for the heart rate monitoring. This method allows the extraction of the heart rate from the RS-peaks frequency by means of the classification of the different peaks (RS-peaks and others) which compose the ECG signal, using the distance between a local maximum followed by a local minimum (amplitude Max-Min) as the feature for clustering (depicted in Fig. $3 \mathrm{c}$ ). Then, the results of the classification allow the high accuracy detection of the R-peaks (Fig. 3d). As can be seen, this method, applied to an extract of $60 \mathrm{~s}$ from a signal recorded
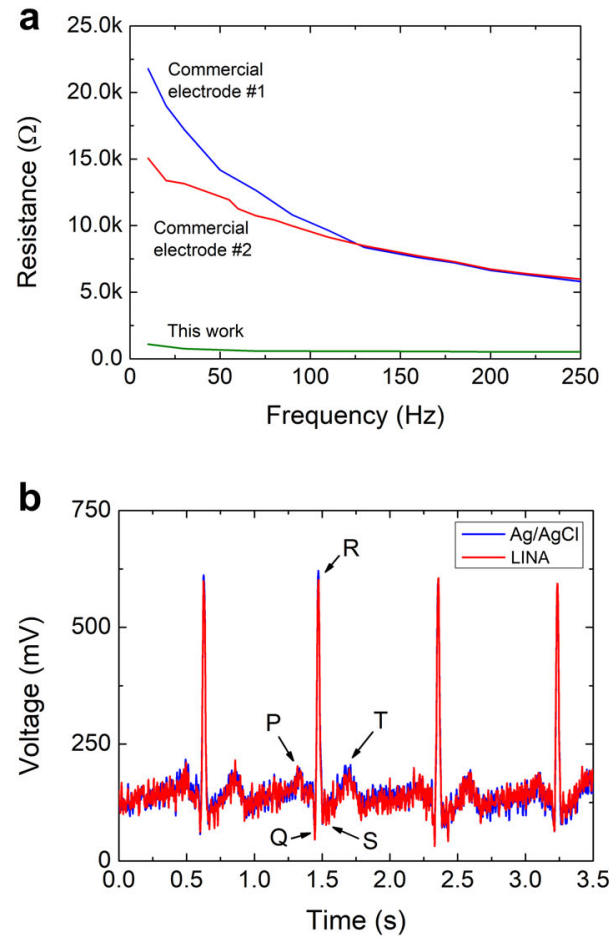

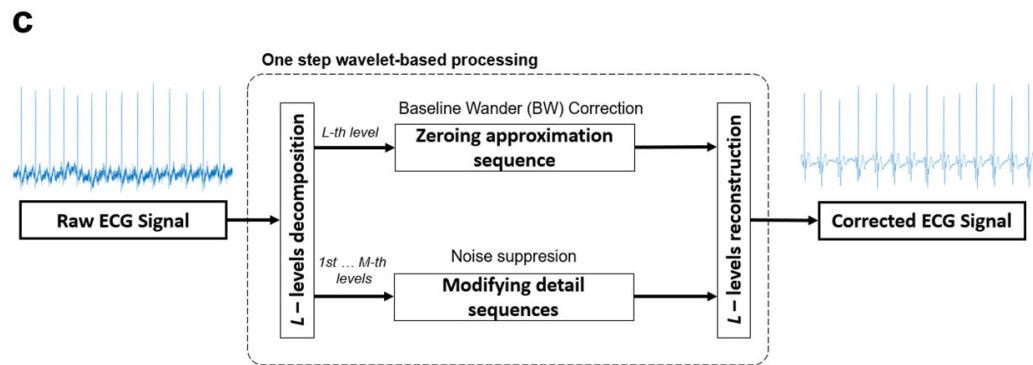

d
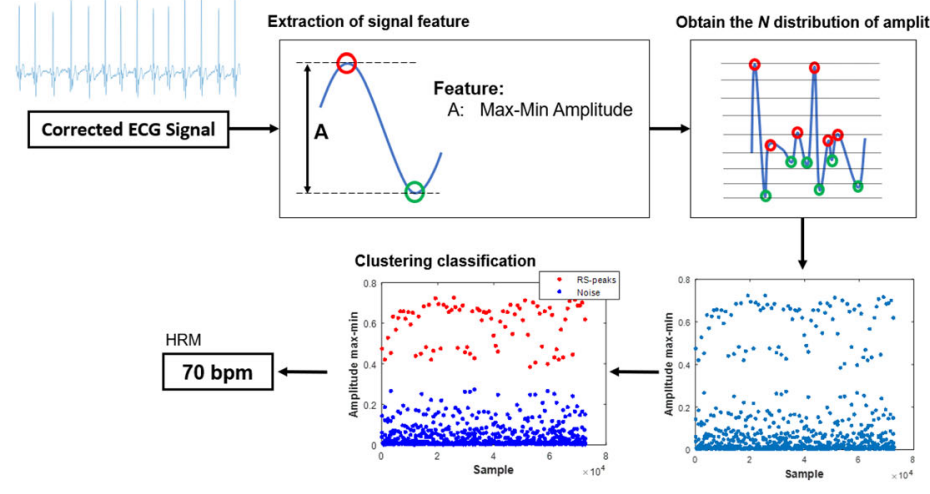

Fig. 2 Electrocardiography recording and processing methods. a Comparison of the skin-electrode contact resistance as a function of the frequency for two commercial electrodes (Ambu ${ }^{\circledR}$ BlueSensor VL and Biopac EL503, respectively) and the LINA electrode. b ECG recordings carried out simultaneously with the LINA electrodes (in red) and the $\mathrm{Ag} / \mathrm{AgCl}$ commercial electrodes (in blue). c One step waveletpreprocessing method for the baseline wandering and noise correction. $\mathbf{d}$ Clustering-based method for the heart rate monitoring based on the max-min amplitude 
a

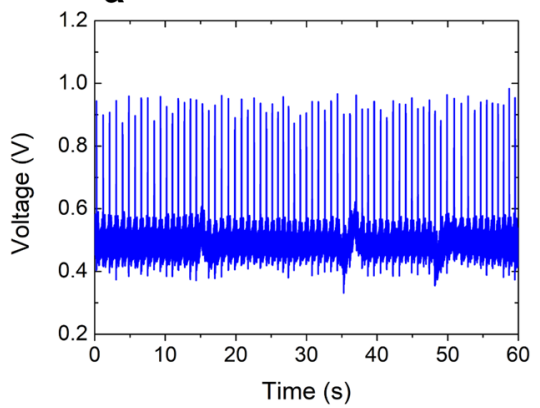

b

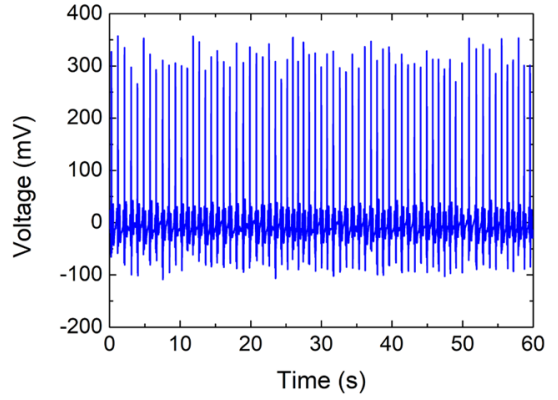

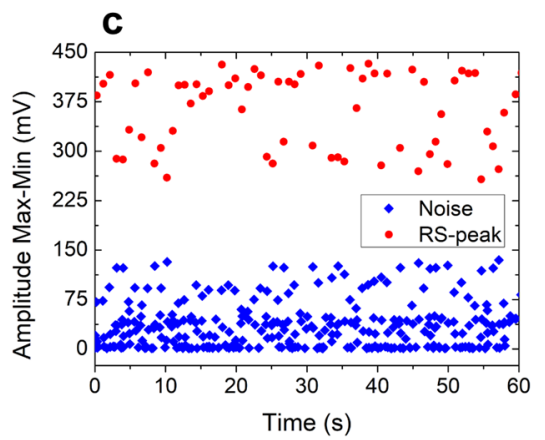
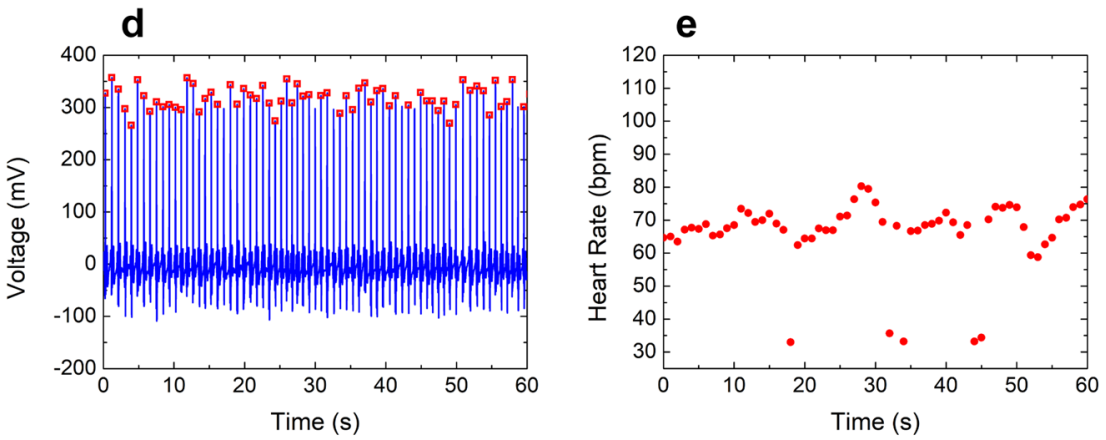

Fig. 3 Electrodes performance in electrocardiography and heart rate monitoring. a Raw ECG signal recording with some motion artifacts performed with the LINA-based electrodes. b Resulting signal after the baseline correction and noise suppression using the proposed DWTbased method. c Two-cluster classification (RS-peaks and noise) of the peaks which compose the ECG signal. d Identification of the RS-peaks along the $60 \mathrm{~s}$ signal from the clustering algorithm results. e Evolution of the heart rate over time extracted from the RS-peaks frequency

with the LINA electrodes, yields a $94.3 \%$ of accuracy, since it allows to detect 66 R-peaks from a total of 70 R-peaks (four false negatives). These results are comparable to that obtained for commercial electrodes. Finally, the frequency of the RS-peaks is used to extract the instantaneous heart rate as shown in Fig. 3e, where the outliers resulting of the false negatives can also be appreciated. Future studies aim to include a next stage to correct these false negatives, as well as to consider alternative machine/ deep learning techniques to automatically extract features from ECG signals. For instance, the approach proposed by Xia et al., ${ }^{29}$ which combines a stacked denoising autoencoder with a softmax regression for the feature extraction and its subsequent classification to detect cardiac arrhythmia, or the one proposed by Zhang et al., ${ }^{30}$ which is based on convolutional neural networks. Alternatively, we aim to combine the electrodes proposed in this work with our recently presented wearable system for biosignal acquisition. $^{31}$

\section{MATERIALS AND METHODS}

Materials

Flexible Kapton ${ }^{\circledR}$ HN films with a thickness of $125 \mu \mathrm{m}$ obtained from DuPontTM were used as raw material for LINA production in our experiments. Ag-based conductive paint was acquired from RS Pro. Two Comercial silver/silver chloride $(\mathrm{Ag} / \mathrm{AgCl}) \mathrm{ECG}$ electrodes were used for comparison, the Ambu ${ }^{\circledast}$ BlueSensor VL (VL-00-S/25, from Ambu A/S) and the EL503 general purpose electrode (from Biopac Systems Inc.), together with the SIGNAGEL ${ }^{\circledR}$ electrolyte gel (15-25 from Parker Laboratories Inc). The laminating pouches Capture 125 acquired from Fellowes Inc. were used to seal and isolate the LIG electrodes. Moreover, the photothermal process was carried out using a DIY CNC laser engraver $(\lambda=405 \mathrm{~nm})$ with adjustable laser power and laser spot size.

\section{Material characterization}

The morphology and structural properties of the LINA layer were analyzed by scanning electron microscopy (SEM), Raman spectroscopy, and X-ray photoelectron spectroscopy (XPS). SEM-images were recorded using a
NVision 40 field-emission scanning electron microscope (from Carl Zeiss) at an extraction and acceleration voltage of $5 \mathrm{kV}$ and a working distance of $6 \mathrm{~mm}$. Raman-spectra were acquired using a JASCO NRS-5100 dispersive micro-Raman spectrometer with an Elforlight G4-30 green diode $(\lambda=$ $532 \mathrm{~nm}$ ) as excitation source. ${ }^{32}$ XPS experiments were performed on a Katros Axis Ultra-DLD spectrometer with an X-ray power of $450 \mathrm{~W}$ in a vacuum chamber with a pressure $10^{-10}$ Torr. CasaXPS software (from Casa Software Ltd.) was used for the C1s spectra decomposition. A 3D profile was also acquired using a DekTak XT profilometer from Bruker. For the electrical characterization of the samples we used the four-point-contact method based on a probe head from Jandel connected to a B2901 A source measuring unit (from Keysight) with a constant current of $0.5 \mathrm{~mA}$. The bending tests were automated using a LabVIEW 2016 (from National Instruments) software controlled setup with a bending speed of $10 \mathrm{~mm} \mathrm{~s}^{-1}$ and a minimum bending diameter of $4 \mathrm{~mm}$.

\section{Measurement of the skin-electrode contact resistance}

The contact resistance of the skin-electrode interface was extracted following a modified version of the setup proposed by Spach et al. ${ }^{33}$ (Supplementary Fig. S3a) for both commercial wet $\mathrm{Ag} / \mathrm{AgCl}$ and LINA electrodes. Three electrodes were placed on the forearm (ventral side) of a person at a distance of $5 \mathrm{~cm}$ from each other. For this particular implementation we used an input voltage of $1 \mathrm{~V}$ with a frequency range from 1 to $250 \mathrm{~Hz}$ referenced to the first electrode. The contact resistance $\left(R_{c}\right)$ was calculated using the equation below, where the input current $\left(i_{i n}\right)$ was monitored through the voltage drop $\left(v_{12}\right)$ on the reference resistor $(r)$, while $v_{23}$ corresponds to the the voltage drop in the contact resistance (considering an ideal differential amplifier).

$R_{\mathrm{c}}=\frac{v_{23}}{i_{\text {in }}}=\frac{v_{23} \cdot r}{v_{12}}=\frac{G_{1} \cdot v_{\mathrm{o}_{2}} \cdot r}{G_{2} \cdot v_{\mathrm{o}_{1}}}$

Due to the inherent variability of the signals recorded from one person to another and the signal amplitude variations associated to the use of the different kinds of electrodes, the monitoring stage (differential amplifiers, gains $G_{1}$ and $G_{2}$, and high-frequency noise suppression) was implemented using a reconfigurable field programmable analog array (FPAA) from Anadigm (AN221E04, Anadigm, Inc.) to obtain a reliable and adaptive analog conditioning signal for each case. In this way, the conditioning 
electronics is simplified within the FPAA device as depicted in Supplementary Fig. S3b.

\section{ECG electrodes performance evaluation}

ECG signals were acquired using the wearable body sensing platform Biosignals Researcher Kit (from PLUX wireless biosignals S.A.) combined with an ECG local differential three-lead electrode (also from PLUX wireless biosignals S.A.) with 16 bit resolution and $1 \mathrm{kHz}$ sampling rate. This wearable device has Bluetooth streaming capability which allowed to send the signals to a computer for their analysis. To compare the performance of both commercial and the LINA proposed electrodes, we analyzed the crest factor of each P-QRS-T complex of the raw recorded signals following equation: ${ }^{34}$

$\mathrm{CF}(\mathrm{dB})=20 \log _{10}\left(\frac{\left|S_{\text {peak }}\right|}{S_{\text {rms }}}\right)$

being $S_{\text {peak }}$ the peak amplitude of the ECG signal (R peak) and $S_{\text {rms }}$ the RMS value of the signal along the $\mathrm{P}-\mathrm{QRS}-\mathrm{T}$ complex.

The main sources of noise of the electrocardiogram signals are the power line interference $\left(50\right.$ or $60 \mathrm{~Hz}$ ) and the motion artifacts. ${ }^{35}$ For the noise suppression of the ECG signals we have applied an algorithm based on DWT digital processing techniques proposed by Castillo et al., ${ }^{36}$ which is widely used to reduce the BW and noise in ECG signals, ${ }^{37,38}$ Moreover, the advantage of the custom algorithm proposed by Castillo et al. lies on its feasibility to be used in portable implementations, such as wearables devices. The algorithm, which has been coded in Matlab ${ }^{\circledR}$ (from The MathWorks, Inc.), follows the scheme shown in Fig. 2c. Then, to obtain the noiseless ECG signal, the raw signal is decomposed into $L$ frequency bands $\left(L<\left[\log _{2}\left(F_{\mathrm{s}} / 2\right)\right]\right.$, being $F_{\mathrm{s}}$ the sampling frequency) to, on one hand, remove the BW by the zeroing approximation of the lowest frequencies and, on the other hand, denoise the signal by the modification of the details of levels 1 to $M$ (with $M<L$ ). The frequency details subbands of levels $M+1$ to $L$, where most of the spectral energy should lie, are preserved to avoid losing possible clinically important components of the signal. ${ }^{39}$ Thus, the BW corrected and denoised ECG signal is reconstructed using the modified details of the levels 1 to $M$, the original details of levels $M+1$ to $L$ and the zeroing approximation of the coefficients of level $L$. For this particular implementation we have decomposed the recorded signal (Fig. 3a) into nine frequency levels $(L=9)$, using the first five levels for the noise suppression $(M=5)$. Therefore, the BW correction is applied to the 9th level, which captures frequencies from 0 to $0.9765 \mathrm{~Hz}$, while the adaptive filtering is applied to the first five levels (range from 15.625 to $500 \mathrm{~Hz}$ ). ${ }^{39}$ Thus, from 0.9765 to $15.625 \mathrm{~Hz}$ the signal is unaltered to preserve the information of the QRS low-frequency bands. ${ }^{40}$ The results of the BW and noise correction in the frequency domain are shown in Supplementary Fig. S4.

Together with the implementation of the DWT detailed above, we have applied a clustering-based method to the recorded signals for the heart rate monitoring. This method is an adaptation of the method recently proposed by Castillo et al. ${ }^{28}$ devoted to the extraction of fetal heart rate from the abdominal ECG signal of the mother. In this case, the heart rate is extracted from the RS-peaks frequency of the P-QRS-T complexes following the procedure shown in Fig. 2d. The RS-peak wave morphology shows a local maximum followed by a local minimum, therefore this pattern is localized along the denoised signal and assigned to one of the $N$ intervals according to the maximum to minimum amplitude. The data is then classified into two different clusters (noise and RS-peaks) using the squared Euclidean distance $D^{2}\left(x_{i}, x_{j}\right)=\left(x_{i}-x_{j}\right)^{2}$ and the $k$-medoids ++ algorithm, which ensures a highly accurate classification and a better performance over threshold-based methods, as it has already been demonstrated in our previous work. ${ }^{28}$

\section{DATA AVAILABILITY}

Data available on request from the corresponding author.

\section{CODE AVAILABILITY}

Code available on request from the corresponding author.

\section{ACKNOWLEDGEMENTS}

This work has been partially supported by the Spanish Ministry of Education, Culture and Sport (MECD) and the European Union through the project TEC2017-89955-P, the predoctoral grants FPU16/01451 and FPU16/04043, and the fellowship H2020-MSCAIF-2017 794885-SELFSENS. Additionally, this work was also supported by the German Research Foundation (DFG) and the Technical University of Munich.

\section{AUTHOR CONTRIBUTIONS}

The research aims and methods were proposed by D.P.M., N.R., F.G.R. and M.B.; the fabrication process was carried out by F.J.R. and A.T.; the structural characterization experiments were carried out by F.J.R. and A.R.; the electrical characterization experiments were handled by F.J.R. and N.R.; E.C. and D.P.M. contributed to the signal recording and analysis. F.J.R., E.C. and N.R. wrote the first draft of the manuscript. Editing and revision were carried out by A.R., M.B., F.G.R., D.P.M. and N.R.

\section{ADDITIONAL INFORMATION}

Supplementary information accompanies the paper on the npj Flexible Electronics website (https://doi.org/10.1038/s41528-019-0056-2).

Competing interests: The authors declare no competing interests.

Publisher's note: Springer Nature remains neutral with regard to jurisdictional claims in published maps and institutional affiliations.

\section{REFERENCES}

1. Domanski, M. J. et al. Next steps in primary prevention of coronary heart disease. J. Am. Coll. Cardiol. 66, 1828-1836 (2015).

2. Buse, J. B. et al. Primary prevention of cardiovascular diseases in people with diabetes mellitus. Circulation 115, 114-126 (2007).

3. Wilkins, E. et al. European Cardiovascular Disease Statistics (European Heart Network, Brussels, Belgium, 2017).

4. De Bacquer, D., De Backer, G., Kornitzer, M. \& Blackburn, H. Prognostic value of ECG findings for total, cardiovascular disease, and coronary heart disease death in men and women. Heart 80, 570-577 (1998).

5. Majumder, S., Mondal, T. \& Deen, M. J. Wearable sensors for remote health monitoring. Sensors 17, 1-45 (2017).

6. Kenry, Yeo, J. C. \& Lim, C. T. Emerging flexible and wearable physical sensing platforms for healthcare and biomedical applications. Microsyst. Nanoeng. 2, 16043 (2016).

7. Castano, L. M. \& Flatau, A. B. Smart fabric sensors and e-textile technologies: a review. Smart Mater. Struct. 23, 053001 (2014).

8. Zama, T. \& Shimada, S. Simultaneous measurement of electroencephalography and near-infrared spectroscopy during voluntary motor preparation. Sci. Rep. 5, 16438 (2015).

9. Baek, J.-Y., An, J.-H., Choi, J.-M., Park, K.-S. \& Lee, S.-H. Flexible polymeric dry electrodes for the long-term monitoring of ECG. Sens. Actuators A 143, 423-429 (2008).

10. Gruetzmann, A., Hansen, S. \& Mller, J. Novel dry electrodes for ECG monitoring. Physiol. Meas. 28, 1375 (2007).

11. Kuniharu, T., Wataru, H., Shingo, H., Takayuki, A. \& Seiji, A. Toward flexible and wearable human-interactive health-monitoring devices. Adv. Healthc. Mater. 4, 487-500 (2014).

12. Misra, V. et al. Flexible technologies for self-powered wearable health and environmental sensing. Proc. IEEE 103, 665-681 (2015).

13. Liu, L., Yu, Y., Yan, C., Li, K. \& Zheng, Z. Wearable energy-dense and power-dense supercapacitor yarns enabled by scalable graphenemetallic textile composite electrodes. Nat. Commun. 6, 7260 (2015).

14. Vargas-Quesada, B., Chinchilla-Rodrguez, Z. \& Rodriguez, N. Identification and visualization of the intellectual structure in graphene research. Front. Res. Metr. Anal. 2, 7 (2017).

15. Celik, N., Manivannan, N., Strudwick, A. \& Balachandran, W. Graphene-enabled electrodes for electrocardiogram monitoring. Nanomaterials 6, 156 (2016).

16. Lou, C. et al. Flexible graphene electrodes for prolonged dynamic ECG monitoring. Sensors 16, 1833 (2016).

17. Karim, N. et al. All inkjet-printed graphene-based conductive patterns for wearable e-textile applications. J. Mater. Chem. C 5, 11640-11648 (2017).

18. Jung, $H$. et al. CNT/PDMS composite flexible dry electrodesfor long-term ECG monitoring. IEEE Trans. Biomed. Eng. 59, 1472-1479 (2012).

19. Jiao, L. et al. Laser-induced graphene on additive manufacturing parts. Nanomaterials 9, 90 (2019). 
20. Romero, F. J. et al. In-depth study of laser diode ablation of kapton polyimide for flexible conductive substrates. Nanomaterials 8, 517 (2018).

21. Romero, F. J. et al. Design guidelines of laser reduced graphene oxide conformal thermistor for loT applications. Sens. Actuators A 274, 148-154 (2018).

22. Duy, L. X. et al. Laser-induced graphene fibers. Carbon 126, $472-479$ (2018).

23. Lin, J. et al. Laser-induced porous graphene films from commercial polymers. Nat. Commun. 5, 5714 (2014)

24. Wu, J.-B., Lin, M.-L., Cong, X., Liu, H.-N. \& Tan, P.-H. Raman spectroscopy of graphene-based materials and its applications in related devices. Chem. Soc. Rev. 47, 1822-1873 (2018).

25. Meziane, N., Webster, J. G., Attari, M. \& Nimunkar, A. J. Dry electrodes for electrocardiography. Physiol. Meas. 34, R47-R69 (2013).

26. Elgendi, M., Mohamed, A. \& Ward, R. Efficient ECG compression and QRS detection for e-health applications. Sci. Rep. 7, 459 (2017).

27. Barold, S. S. Willem einthoven and the birth of clinical electrocardiography a hundred years ago. Card. Electrophysiol. Rev. 7, 99-104 (2003).

28. Encarnacion Castillo, D. P. M., Garcia, A., Parrilla, L., Ruiz, V. U. \& Alvarez-Bermejo, J. A. A clustering-based method for single-channel fetal heart rate monitoring. PLoS ONE 13, e0199308 (2018).

29. Xia, Y. et al. An automatic cardiac arrhythmia classification system with wearable electrocardiogram. IEEE Access 6, 16529-16538 (2018).

30. Zhang, Q., Zhou, D. \& Zeng, X. Heartid: a multiresolution convolutional neural network for ecg-based biometric human identification in smart health applications. IEEE Access 5, 11805-11816 (2017).

31. Toral, V. et al. Wearable system for biosignal acquisition and monitoring based on reconfigurable technologies. Sensors 19, 1590 (2019).

32. Ferrari, A. \& Basko, D. Raman spectroscopy as a versatile tool for studying the properties of graphene. Nat. Nanotechnol. 8, 235-246 (2013).

33. Spach, M. S., Barr, R. C., Havstad, J. W. \& Long, E. C. Skin-electrode impedance and its effect on recording cardiac potentials. Circulation 34, 649-656 (1966).

34. Takamatsu, S. et al. Direct patterning of organic conductors on knitted textiles for long-term electrocardiography. Sci. Rep. 5, 15003 (2015).
35. Satija, U., Ramkumar, B. \& Manikandan, M. S. Automated ECG noise detection and classification system for unsupervised healthcare monitoring. IEEE J. Biomed. Health Inform. 22, 722-732 (2018).

36. Castillo, E. et al. Efficient wavelet-based ECG processing for single-lead FHR extraction. Digit. Signal Process. 23, 1897-1909 (2013).

37. Mamaghanian, H., Khaled, N., Atienza, D. \& Vandergheynst, P. Compressed sensing for real-time energy-efficient ECG compression on wireless body sensor nodes. IEEE Trans. Biomed. Eng. 58, 2456-2466 (2011).

38. Sharma, L., Dandapat, S. \& Mahanta, A. ECG signal denoising using higher order statistics in wavelet subbands. Biomed. Signal Process. Control 5, 214-222 (2010).

39. Castillo, E. et al. Noise suppression in ECG signals through efficient one-step wavelet processing techniques. J. Appl. Math. 2013, 1-13 (2013).

40. Zidelmal, Z., Amirou, A., Adnane, M. \& Belouchrani, A. QRS detection based on wavelet coefficients. Comput. Methods Prog. Biomed. 107, 490-496 (2012).

(i) Open Access This article is licensed under a Creative Commons BY Attribution 4.0 International License, which permits use, sharing, adaptation, distribution and reproduction in any medium or format, as long as you give appropriate credit to the original author(s) and the source, provide a link to the Creative Commons license, and indicate if changes were made. The images or other third party material in this article are included in the article's Creative Commons license, unless indicated otherwise in a credit line to the material. If material is not included in the article's Creative Commons license and your intended use is not permitted by statutory regulation or exceeds the permitted use, you will need to obtain permission directly from the copyright holder. To view a copy of this license, visit http://creativecommons. org/licenses/by/4.0/.

(c) The Author(s) 2019 